\title{
COLOUR PHOTOGRAPHY OF VISUAL PURPLE SOLUTIONS*
}

BY

\author{
H. J. A. Dartnall
}

From the Vision Rcsearch Unit, Medical Research Council, Institute of Ophthalmology

For a recent spectrographic investigation of the light-sensitive retinal pigment visual purple, the results of which will be published elsewhere, it was necessary to prepare solutions of visual purple on a larger scale than has been attempted previously. Generally, about 100 dark-adapted frogs (rana temporaria) were used to prepare 50 to, $70 \mathrm{ml}$. of solution. The extraction procedures

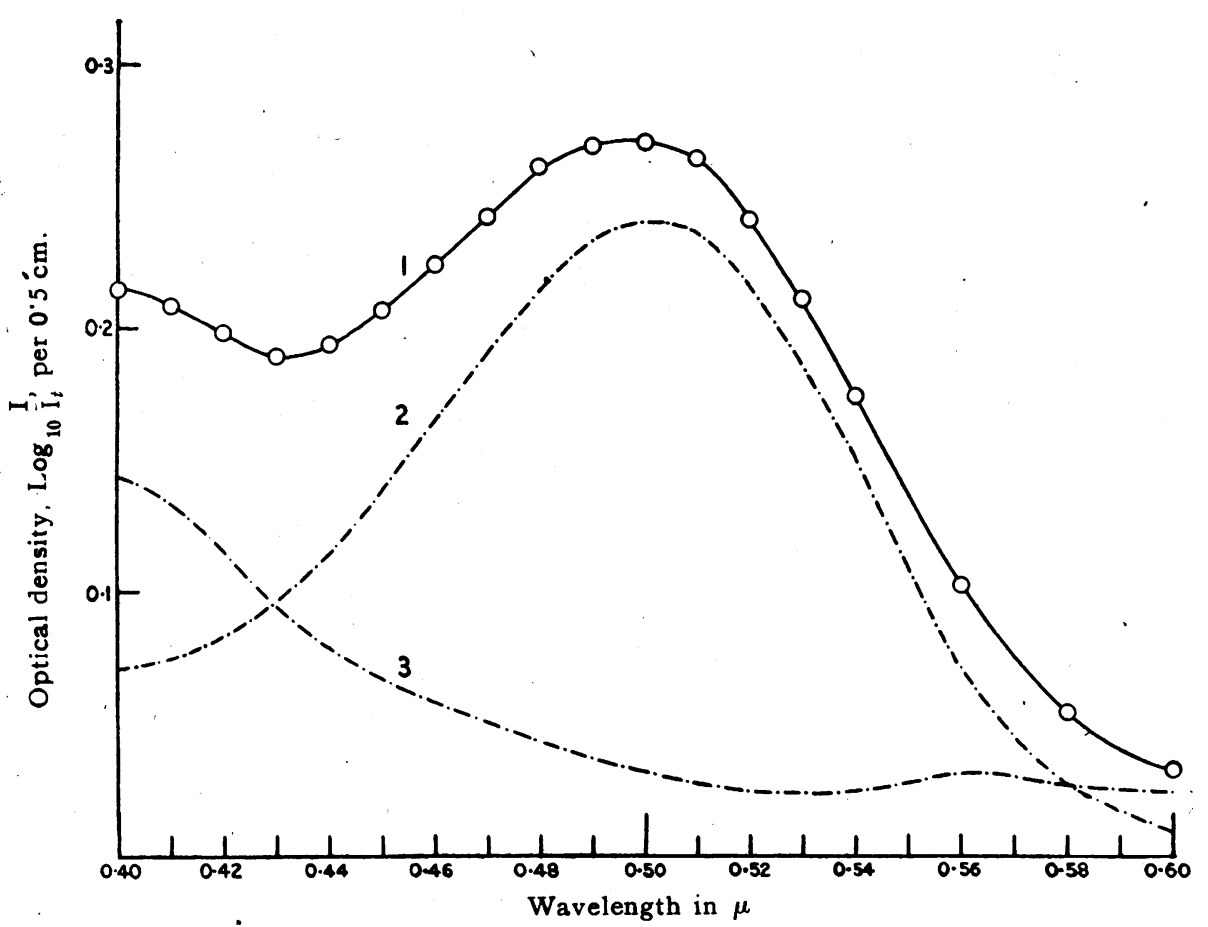

FIGURE.-Curve 1, the absorption spectrum of the unbleached solution; curve 2 , the absorption of the contained visual purple: curve 3 , the residual absorption due to impurities.

were conducted partly in dim red light and, where possible, in darkness.

The opportunity was taken of obtaining colour photographs of

* Received for publication September 12, 1949. 
an unbleached and bleached solution. The photographs were taken by $\mathrm{Mr}$. S. E. Veronique using Dufaycolor cut film and, as illuminant, a Phillip's P.F.56 flash-bulb in conjunction with the appropriate D2/5 gelatine filter. The conditions for obtaining correct colour values were ascertained by preliminary photography of a similar flask containing a dilute potassium permanganate solution.

Plate I shows the appearance of the solution immediately after withdrawal of the flask from its light-tight container. The solution, which is nearly free from opalescence and slightly frothy due to the presence of the surface active digitonin used as extractive, is reddish and not purple in colour. This is partly due to the presence of yellow substances which are extracted together with the visual purple by the digitonin. The effect of absorption by these impurities may be gauged from the Figure which, in addition to showing the total absorption of the solution, shows the respective contributions of visual purple and impurities. No attempt was made to remove these impurities since these crude digitonin extracts are regarded as preferable material for spectrum analysis to purified solutions, in the preparation of which there is the risk of contamination by trace impurities in the chemicals used.

The solution was bleached by exposing it for two minutes to a 100 -watt pearl lamp with reflector at a distance of 18 inches. A photograph of the freshly bleached solution, the $p \mathrm{H}$ of which was about 6, is shown in Plate II. The deep yellow colour is due to transient orange and indicator yellow, formed as a result of the photodecomposition of visual purple. The modifying influence of the constant absorption of the yellow impurities is, of course, much less marked in the bleached solution, since the absorption of the bleached products is very intense in the same spectral region. 


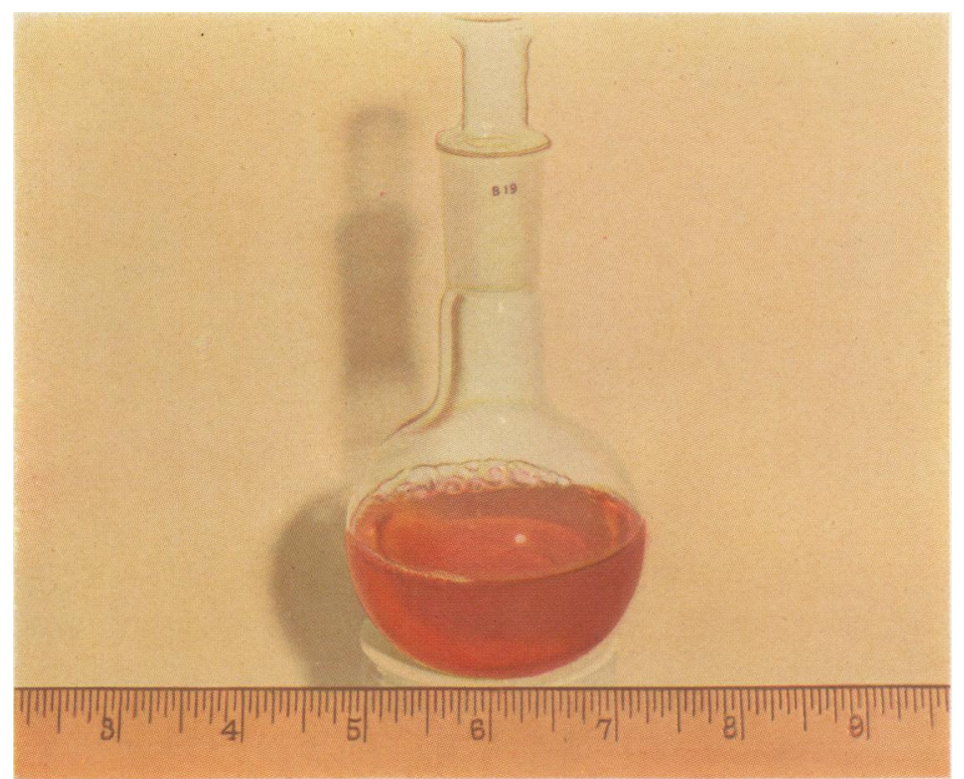

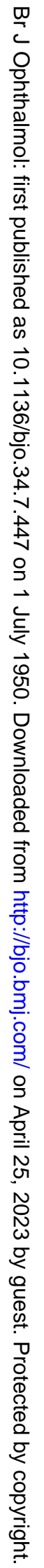

Plate II 